\title{
Facharbeit, Berufe und berufliche Arbeitsmärkte
}

Ist das duale System der Berufsausbildung mit seinen standardisierten Berufsbildern ein Auslaufmodell, das den Übergang in die Wissensgesellschaft behindert, oder ist es das Geheimnis der deutschen Wettbewerbsfähigkeit? Die Antwort hängt zum einen davon ab, wie reformwillig die das System tragenden Sozialpartner sind. Zum anderen ist relevant, ob die Berufsbilder und die Ausbildungsformen Handlungsfähigkeit in neuen Formen flexibler Arbeitsorganisation vermitteln und den Arbeitsplatzwechsel im internen und externen Strukturwandel erleichtern. ${ }^{1}$

GERHARD BOSCH

\section{Einleitung}

Industrie- ebenso wie auch Dienstleistungsarbeit hat sich in den letzten Jahrzehnten grundlegend geändert. In neuen flexibleren Organisationsformen wurden Hierarchieebenen verringert und Koordinationsaufgaben auf die ausführende Ebene verlagert. Der funktionale Aufgabenzuschnitt ist vielfach durch eine prozessorientierte Arbeitsorganisation ersetzt worden, in der Beschäftigte meist im Team mit unterschiedlichen Berufen arbeiten (Spöttl/Blings 2011, S. 19). Betriebliche Reorganisationsprozesse erfordern zudem häufig einen Arbeitsplatzwechsel im Unternehmen oder in andere Unternehmen. Oft geht auch die Initiative zu einem Tätigkeits- oder Unternehmenswechsel von den Beschäftigten aus, die sich beruflich verbessern wollen oder aus privaten Gründen einen neuen Arbeitsplatz suchen. Die Anforderungen an eine zukunftsträchtige Berufsausbildung lassen sich daher nicht alleine aus der Entwicklung der Arbeitsprozesse ableiten, sondern ergeben sich auch aus dem zu erwartenden internen und externen Strukturwandel sowie der erwarteten und gewünschten Mobilität im Erwerbsverlauf der Beschäftigten.

$\mathrm{Ob}$ das deutsche System der dualen Berufsausbildung die Anforderungen an eine nachhaltige Ausbildung noch hinreichend erfüllt, ist seit langer Zeit Gegenstand heftiger Debatten. Die Mehrheit der deutschen Berufsbildungsforscher sieht den Niedergang des Berufskonzepts und der dualen Berufsausbildung eingeläutet. Das Konzept des „Lebensberufs“ gilt als „historisches Relikt“ (Lipsmeier 1998). Voss spricht von einer Auflösung von Berufsbildern und der Entwicklung von „Individualberufen“ (Voss 1998, S. 484). Baethge et al. (2007) sehen Nachteile im erfahrungsorientierten Lernen, da man in der Wissensgesellschaft mehr theoretisches Wissen brauche, welches am besten in besonderen Bildungseinrichtungen vermittelt werde. Das Berufskonzept wird in diesen Erosionsszenarien allerdings statisch gefasst, sodass jeder Wandel zwangsläufig als Niedergang interpretiert werden muss. Zudem fehlen die Akteure, die auf Probleme im Berufsbildungssystem reagieren können. Die wichtigsten Akteure, nämlich Gewerkschaften und Arbeitgeberverbände, finden allenfalls Erwähnung als angebliche Verteidiger des Alten ohne jedes Interesse an einer Modernisierung.

Im Folgenden soll der Frage nachgegangen werden, welche Rolle berufliche Bildung bei der Bewältigung des internen und externen Strukturwandels für Beschäftigte und Unternehmen spielen kann. Dazu soll zunächst gefragt werden, ob Berufe und berufliche Arbeitsmärkte ge-

1 Dieser Beitrag ist eine Ausarbeitung der Keynote auf der Tagung der Arbeitsgemeinschaft Berufsbildungsforschungsnetz (AG BFN) zum Thema "Weiterentwicklung von Berufen" im Bundesinstitut für berufliche Bildung (BIBB) am 17/18.4.2012 in Bonn. 
eignete Institutionen für flexible Unternehmen und Arbeitsmärkte sind (Abschnitt 2). Anschließend soll der Fachkräfteeinsatz in unterschiedlichen Segmenten des deutschen Arbeitsmarktes zwischen 1995 und 2007 nachgezeichnet werden (3). Zum Abschluss wird untersucht, ob der hiesige und infolge des starken dualen Systems im internationalen Vergleich geringe Akademikeranteil ein Wettbewerbsnachteil ist (4).

\section{Berufe und berufliche Arbeitsmärkte}

Im Folgenden wird zunächst der Idealtypus beruflicher Arbeitsmärkte umrissen, um die Funktionsbedingungen dieses Arbeitsmarktsegments besser zu verstehen (2.1). Da angesichts des raschen Strukturwandels spezifische Berufe schnell obsolet werden, mussten breitere und dynamischere Berufsbilder entwickelt werden. Diese neuen Berufskonzepte sind Gegenstand von Abschnitt 2.2. Anschließend wird dargelegt, warum eine reformierte Beruflichkeit Beschäftigungsfähigkeit und Flexicurity nachhaltig sichert (2.3).

\subsection{Der Idealtypus beruflicher Arbeitsmärkte}

Berufliche Arbeitsmärkte konstituieren sich über standardisierte, über den einzelnen Betrieb transferierbare Qualifikationen (Sengenberger 1987, S. 126ff.). Die Qualifikationen werden in Berufsbildern gebündelt. Die Anerkennung der beruflichen Qualifikationen unterliegt einer überbetrieblichen Institution, die auch Inhalte, Ausbildungsabläufe und Qualifikationsnachweise in Form von Zertifikationen (wie etwa Facharbeiterbrief, Diplom, Bachelor- oder MasterAbschluss) festlegt. Um Anerkennung auf dem Arbeitsmarkt zu finden, müssen die in Berufen geordneten Qualifikationsbündel von einer kritischen Masse von Arbeitgebern nachgefragt werden. Ohne eine gewisse Nachfragedichte wird sich kein durchsetzungsfähiger Wunsch nach einer überbetrieblichen Standardisierung der Qualifikationen entwickeln können. Die Nachfragedichte kann durch eine Verbreiterung der Ausbildung erhöht werden. Bei unzureichender Nachfragedichte auf regionaler Ebene kann überregionale Mobilität erforderlich sein.

Im Idealfall entspricht die Arbeitsplatzstruktur dem Zuschnitt der Berufe. In den dualen Ausbildungssystemen ist dies schon bei der Ausbildung Funktionsvoraussetzung, da nur dann auch Ausbildungsinhalte praxisnah vermittelt werden können. Durch eine kontinuierliche Modernisierung der Berufsbilder können auch Innovationsprozesse in den Betrieben angestoßen werden. Das gilt insbesondere für kleine und mittlere Unternehmen ohne eigene Forschung und Entwicklung, in denen Innovationsprozesse vorrangig von der Aus- und Weiterbildung der Schlüsselpersonen getragen werden. Dies gelingt allerdings in wenig innovativen Betrieben nur, wenn die Anstöße zur Moder- nisierung von außen über die Berufsschulen oder überbetriebliche Ausbildungszentren kommen.

Berufe sind verlässliche Signale für die erforderlichen Qualifikationen in bestimmten Tätigkeitsfeldern, sodass Arbeitsplatzwechsel und Einstellungen ohne oder nur mit geringen Anlernkosten möglich sind. ${ }^{2}$ Dies erleichtert zudem den externen Strukturwandel und erhöht die Freiheitsgrade der Beschäftigten auf dem Arbeitsmarkt, die im Idealfall ohne Qualifikations- und Einkommensverluste zu einem anderen Unternehmen wechseln können.

$\mathrm{Zu}$ geschlossenen beruflichen Arbeitsmärkten haben nur Personen mit den geforderten Qualifikationen Zutritt, wie etwa zu den gefahrengeneigten Handwerksberufen durch das Meisterprivileg oder in den angelsächsischen Ländern durch eine qualifikationsgebundene Zulassung (Licensing). Weitere Beispiele sind vor allem Gesundheitsberufe und freie Berufe wie der des Rechtsanwaltes oder Notars. $\mathrm{Zu}$ offenen beruflichen Arbeitsmärkten hingegen haben auch Beschäftigte mit einer fachfremden oder ohne Berufsausbildung Zugang. Sie können sich die fehlenden Qualifikationen durch Anlernen erwerben. Angelernte mögen nach langer Betriebszugehörigkeit und entsprechend ausreichender Berufspraxis über die gleichen Qualifikationen wie Fachkräfte verfügen. Allerdings können sie ihre Qualifikationen nicht gleichwertig dokumentieren, was bei unfreiwilligem Arbeitsplatzwechsel zu Abstufungen und Lohneinbußen führt (Bosch 1978).

Berufe sind komplexe soziale Konstrukte, die in mehreren Institutionen zugleich verankert sind. Veränderungsdruck kann aus allen diesen Institutionen kommen. Im strukturellen Wandel verschwinden Berufe und neue müssen entworfen werden. Darüber hinaus können sich die Arbeitsplatzzuschnitte und Anforderungen in einem Berufsfeld so verändern, dass die alten Berufe dysfunktional werden. Die Nachfrage kann dann nur mit modernisierten Berufsbildern wiederbelebt werden. Auf der Angebotsseite können Zertifikate ihre Signalfunktion verlieren, wenn die Ausbildung nicht mehr ihr Qualitätsversprechen erfüllt und die Kontrollinstanzen, die Mindeststandards sichern, versagen. Dann werden sich die Unternehmen an anderen Signalen, wie etwa dem Renommee der Ausbildungseinrichtungen, den Schulabschlüssen, der Herkunft etc., orientieren. Im Idealtyp beruflicher Arbeitsmärkte spielen diese anderen Signale keine Rolle. In der Realität jedoch haben sie eine große Bedeutung, insbesondere bei hoher Arbeitslosigkeit, wenn die Unternehmen die Bewerber nach zusätzlichen Kriterien sortieren können.

Schließlich müssen die Institutionen, die die Berufsausbildung tragen, durch starke Akteure gestützt und weiter-

2 Gerade weil Berufe auf viele unterschiedliche Tätigkeiten vorbereiten, führt es nur zur Verwirrung, wenn man diese Tätigkeiten, die sich zudem schneller ändern als Berufe, als Erwerbs- (Petersen 2012) oder Erwachsenenberufe (Pahl 2010) bezeichnet. 
entwickelt werden. Duale Berufsbildungssysteme etwa sind auf reformwillige und verpflichtungsfähige Arbeitgeberverbände und Gewerkschaften angewiesen, die nicht nur gemeinsam die Modernisierung der Berufsbildung, sondern zugleich auch die Ausbildungsbereitschaft der Unternehmen sowie die angemessene Entlohnung der Fachkräfte sichern. In einigen akademischen Berufen sind die Selbstständigen in berufsständische Organisationen eingebunden, die nicht alleine bestimmte Berufsdomänen verteidigen, sondern auch - wie bei den „verkammerten“ Berufen - soziale Sicherungssysteme aufbauen, die jeweiligen Einkommensinteressen vertreten, die autonome Berufsausübung verteidigen und auch berufsspezifische Wertekanons formulieren. In der soziologischen Theorie spricht man von Professionalisierung, die Daheim (1967, S.42ff.) als Verfeinerung, Anhebung, Aufwertung einer Berufsposition bezeichnet hat. Selbststeuerung, hohes systematisiertes Wissen und berufliche Autonomie gelten als Merkmale von Professionen, die sich damit von den „Industrieberufen“ mit ihrer Weisungsgebundenheit, Verankerung in Erfahrung und der Fremdbestimmung abheben sollen (Hartmann 1968). Mit der Massenproduktion akademischer Qualifikationen und der Anhebung der Anforderungen in der Berufsausbildung sind diese Unterschiede jedoch fließender geworden.

In der Literatur zu beruflichen Arbeitsmärkten wurde die Bedeutung der Lohnstrukturen und Sozialsysteme für die Konstitution beruflicher Arbeitsmärkte bislang kaum beachtet. Im Idealfall sind Produktivität und Löhne aller Beschäftigten eines Berufes gleich, sodass die überbetriebliche Mobilität nicht durch Transaktionskosten beeinträchtigt wird. Bei Arbeitgebermacht können jedoch qualifizierte Beschäftigte auch unterhalb ihrer Produktivität entlohnt werden, und es können sich unabhängig von der Nachfragesituation hohe Lohnunterschiede für die gleichen Berufe verfestigen, was die Mobilitätsbereitschaft der Fachkräfte sowie die Ausbildungsbereitschaft Jugendlicher stark beeinträchtigen kann. Überbetriebliche standardisierte Lohnstrukturen, etwa über Flächentarife, die auch den Abstand zu nicht qualifizierter Arbeit sichern, verbessern somit die Funktionsbedingungen von beruflichen Arbeitsmärkten erheblich. Das gilt ebenso für die soziale Sicherung. Überbetriebliche soziale Sicherungssysteme garantieren die Portabilität der Ansprüche bei einem Betriebswechsel, während betriebliche Sozialleistungen oft nicht mitgenommen werden können und die Betriebsbindung erhöhen. Diese sozial- und lohnpolitischen Voraussetzungen des Funktionierens beruflicher Arbeitsmärkte rücken erst in den letzten Jahren durch eine Erosion der Tarifverträge in vielen Branchen ins Bewusstsein.

In der Realität sind Mischformen beruflicher mit betriebsinternen und unstrukturierten Arbeitsmärkten typisch. Betriebsinterne Arbeitsmärkte basieren auf langer Betriebszugehörigkeit und interner Rekrutierung für alle Aufstiegspositionen. Unstrukturierte Arbeitsmärkte finden sich bei einfachen Tätigkeiten und geringer wechselseitiger Bindung der Beschäftigten und Unternehmen. Interne Ar- beitsmärkte können berufliche Arbeitsmärkte sowohl ersetzen als auch vereinnahmen. Betriebliche Anlernprozesse können eine Berufsausbildung teilweise ersetzen, was bei komplexeren Anforderungen allerdings nur bei langer Betriebszugehörigkeit möglich ist. Bei der Vereinnahmung werden beruflich Beschäftigte durch übertarifliche Löhne oder betriebliche Sozialleistungen an den Betrieb gebunden und das berufliche Lernen durch mehrfache Unternehmenswechsel entfällt. Welche Auswirkungen eine lange Betriebszugehörigkeit auf die beruflichen Qualifikationen hat, hängt allerdings von der betrieblichen Arbeitsorganisation und den Karriereprozessen ab. Bei sehr spezialisierten Produkten und Verfahren und der Zuweisung von Routinearbeiten kommt es auf Dauer zu einer Dequalifikation. Bei breitem Zuschnitt und häufigem Wechsel der Aufgaben sowie Teilnahme an Weiterbildung, innerbetrieblichem Arbeitsplatzwechsel und betrieblichen Karrieren hingegen werden die beruflichen Fähigkeiten nicht nur erhalten, sondern ähnlich wie bei Unternehmenswechseln sogar erweitert. Die Internalisierung ist umso leichter möglich, je weniger die Bezahlung und soziale Sicherung durch überbetriebliche Lohninstitutionen und Sozialsysteme standardisiert wird. Besonders überdurchschnittlich produktive Betriebe können so ihre Belegschaft vor der Abwerbung durch externe Konkurrenz schützen.

Die andere Mischform findet sich in Überlappungen zwischen beruflichen und den unstrukturierten Arbeitsmärkten für Un- und Angelernte. Bei einem Überangebot an beruflich Qualifizierten können Unternehmen beruflich Qualifizierte anstelle von An- und Ungelernten einstellen. Eine berufliche Qualifikation gilt als Signal für extrafunktionale Qualifikationen, wie Pünktlichkeit, Zuverlässigkeit, Allgemeinbildung, Kommunikationsfähigkeit etc., die in diesem Segment beim Arbeitsangebot nicht ohne Weiteres vorausgesetzt werden können. Die „Verberuflichung“ unstrukturierter Arbeitsmärkte ist am ehesten bei einem Überangebot qualifizierter Kräfte, bei einem Lohnverfall in beruflichen Arbeitsmärkten und bei hoher Produktivität der Anlerntätigkeiten, die überdurchschnittliche Löhne ermöglicht (so bspw. bei Fließbandarbeit in Großbetrieben) zu beobachten. Diese Überschneidungszonen sind Gegenstand von Abschnitt 4.

\subsection{Grund-, Basis-, Kernberufe und Berufsfamilien}

In der Beschreibung des Idealtypus beruflicher Arbeitsmärkte sind Berufe zunächst noch abstrakt als überbetrieblich strukturiertes Qualifikationsbündel gefasst worden. Das reicht natürlich nicht aus, um die aktuellen und zukünftigen Anforderungen an konkrete Berufsbilder zu beschreiben, die sich in den vergangenen Jahrzehnten drastisch verändert haben.

Bis zur Verabschiedung des Berufsbildungsgesetzes wurde überwiegend in spezialisierten Monoberufen mit Ausbildungsgängen, die oft noch aus den 1930er Jahren 
stammten, ohne innere Differenzierung ${ }^{3}$ ausgebildet. In den Zeiten stabilen Wirtschaftswachstums mit hoher Betriebsbindung war die hohe Spezialisierung der Beschäftigten und ihre geringe funktionale Flexibilität kein großes Problem. Durch die Integration vormals getrennter Prozesse sowie neue Arbeitskonzepte entstanden jedoch umfassendere Aufgabenbündel, sodass die kleinteiligen Berufe nicht mehr die neuen Aufgabenzuschnitte widerspiegelten. Standortkonzentrationen und Betriebsstilllegungen erforderten zudem überbetriebliche Mobilität. In der Industriesoziologie wurden neben den engen Berufsbildern auch die Entwertung von beruflichen Qualifikationen in betrieblichen Routinetätigkeiten und die auf dem Arbeitsmarkt nicht dokumentierbaren Anlernqualifikationen als zentrale soziale Risiken identifiziert (Böhle/Altmann 1972, S. 26ff.; Bosch 1978), auf die man mit einer anderen Qualifizierungspolitik reagieren müsse.

Seit Ende der 1960er Jahre wurde daher die Notwendigkeit neuer Berufskonzepte diskutiert, für die unterschiedliche Begriffe gefunden wurden. Man sprach von „Grundberufen“ (Ulrich/Lahner 1970, S. 41), von „offenen dynamischen Berufsbildern“ (Heidegger/Rauner 1997, S. 20ff.) oder „Kernberufen“ (Spöttl/Blings 2011). Während es anfangs nur um die Zusammenfassung von Berufen ging, thematisierte man zunehmend auch die Öffnung starrer Curricula für das Lernen in betrieblichen Prozessen.

Entlang dieser Grundgedanken wurde die duale Berufsbildung modernisiert. Viele sich überlappende und angrenzende Berufe wurden zusammengefasst. Anfang der 1970er Jahre vereinbarten die Sozialpartner z. B. in der Bauwirtschaft, deren Arbeitsprozesse durch die Koordination unterschiedlicher Gewerke geprägt sind, für 16 Berufe eine gemeinsame Grundausbildung im ersten Jahr und eine dann teilspezialisierte, aber immer noch berufsübergreifende

ABB. 1

\section{Grundformen didaktischer Ansätze in der Ausbildung}

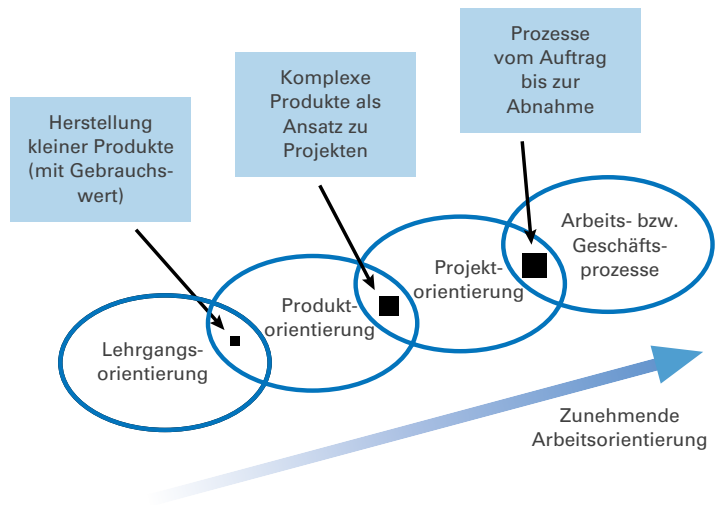

Quelle: Darstellung des Autors auf der Basis von Hahne (1999, S. 205).
Fachausbildung im zweiten Jahr, bevor im dritten Jahr die Spezialisierung auf einen Beruf erfolgte. Dieses Grundprinzip war wegweisend für Reformen in anderen Berufsdomänen. 1987 wurden 47 Metallberufe zu 16 Berufen mit gemeinsamer Grundausbildung und anschließender Spezialisierung ausgehandelt. In einem weiteren Reformschritt wurden 2004 die 16 Metallberufe zu fünf Kernberufen zusammengefasst, in denen zu 50 \% gemeinsame Kernqualifikationen über die gesamte Ausbildungszeit vermittelt werden. Die Dienstleistungs- und Produktionstätigkeiten übergreifenden neuen vier IT-Berufe aus dem Jahr 1997 mit gemeinsamen Kernqualifikationen standen Pate bei der letzten Reform in der Metallindustrie. In anderen Berufen vereinbarte man Wahlbausteine, die in der Berufsausbildung oder später in der Weiterbildung erlernt werden konnten. Vergleichbare Strukturierungsprinzipien finden sich heute in vielen Berufen (Brettschneider/Schwarz 2011, S. 45).

Nicht überall gelang die Zusammenlegung von Berufen. So überschneiden sich die über 50 Büroberufe in großen Teilen und könnten zu wenigen Kernberufen mit Möglichkeiten einer Spezialisierung zusammengefasst werden. Dagegen stehen aber starke Interessen aus vielen Teilbranchen. Abgesehen von reformhinderlichen Spezialinteressen spricht ein Argument für die Beibehaltung von Branchenberufen. Wenn die Unternehmen einer Branche ,ihren“von ihnen selbst gestalteten eigenen Beruf haben, erhöht dies die Bereitschaft, in diesem Beruf auszubilden und sich für seine Modernisierung verantwortlich zu fühlen. In diesem Fall bietet sich eine Vereinheitlichung der Grundinhalte in Berufsfamilien unter Beibehaltung der alten Berufsnamen als Alternative an (Brötz et al. 2008), sozusagen eine Modernisierung in alten „Gemäuern.“

Die Modernisierung der Berufsbilder nahm in der Vergangenheit viel Zeit in Anspruch. Die erste Reform der Metallberufe in den 1980er Jahren dauerte ungefähr sieben Jahre. Als Anfang der 1990er Jahre die deutschen Unternehmen mit Einführung der „lean-production“ in kurzer Zeit völlig reorganisiert wurden, einigten sich die Sozialpartner 1995 auf eine Beschleunigung der Neuordnungsverfahren, die bei alten Berufen nicht mehr als ein Jahr und bei neuen Berufen nicht mehr als zwei Jahre dauern sollten. Damit gelang es, das Modernisierungstempo deutlich zu erhöhen. Zwischen 1996 und 2008 wurden 82 neue Berufe geschaffen und 219 bestehende grundlegend modernisiert (Brötz 2009).

Auch die Lernformen haben sich verändert. Grundlegendes Gestaltungsprinzip ist heute das Lernen in typischen Geschäftsprozessen, das Schlüsselqualifikationen wie die Kooperation mit anderen Fachkräften und die Kommuni-

3 Eine britische Expertenkommission äußerte sich in den 1980er Jahren nach einem Besuch in Deutschland sehr kritisch über den engen Zuschnitt der Metall-Berufe und die unzureichende Berücksichtigung neuerTechnologien in den Ausbildungsplänen, vgl. o. A. (1983), S. $25 \mathrm{ff}$. 
kation mit den internen oder externen Kunden bei Auftragsannahme und Ablieferung einschließt. Der frühere enge Lernhorizont, der sich auf ein bestimmtes Produkt oder partielle Produktionsabschnitte beschränkte, wird damit beträchtlich erweitert (Abbildung 1).

\subsection{Flexicurity, Employability, Kompetenzen und Beruflichkeit}

Mit der Entwicklung der europäischen Beschäftigungsstrategie formulierte die Europäische Kommission gemeinsame Orientierungspunkte für eine aktivierende Arbeitsmarktpolitik ihrer Mitgliedsländer. Die zentralen Konzepte sind unter den Stichworten „Employability“ (Beschäftigungsfähigkeit) und „Flexicurity“ (Flexibiliät und Sicherheit) bekannt, die höchst unterschiedlich ausgelegt werden. In der europäischen Debatte um Beschäftigungsfähigkeit findet man die ganze Spannbreite von kurzfristiger Verwertbarkeit bis hin zu nachhaltiger Beschäftigungsfähigkeit im Erwerbsverlauf. „Be acceptable to an employer“ (Mangum 1976, S. 18) ist ein Beispiel für die kurzfristige Perspektive; für die langfristige steht ,the capacity to be productive and to hold rewarding jobs" (McKenzie/Wurzburg 1998, S. 13). Aus beiden Positionen folgen völlig gegensätzliche Politiken. Brussig/Knuth (2009) sehen die Berufsausbildung als wichtigen Baustein für eine nachhaltige Integration von Arbeitslosen.

Ähnlich breit streuen die Auffassungen in der Auslegung von „Flexicurity“. Wichtige Protagonisten der Idee betonen, dass ein ausgebautes System der beruflichen Aus- und Weiterbildung inner- und überbetriebliche Mobilität erleichtern kann (Bekker/Wilthagen 2008, S. 70). Es finden sich aber auch Auslegungen, die durch den Abbau des Kündigungsschutzes den externen Strukturwandel ohne ausreichende soziale Sicherung forcieren wollen.

Der Kompetenzbegriff als Ordnungselement in der Berufsbildung ist im Rahmen der Debatten um den Europäischen Qualifikationsrahmen (EQR) eingeführt worden. Die EU-Mitgliedstaaten haben sich verpflichtet, sämtliche Qualifikationsniveaus der allgemeinen und beruflichen sowie der akademischen Aus- und Weiterbildung acht Referenzniveaus zuzuordnen, um sie auf diese Weise über die Grenzen hinweg vergleichen und die Mobilität erhöhen zu können. Dabei sollen die Qualifikationen nicht anhand von Bildungsgängen und der Abschlüsse, sondern in Form von Lernergebnissen (Outcomes), einschließlich der informell erworbenen, beschrieben werden, die nach Kenntnissen, Fertigkeiten und Kompetenzen unterschieden werden (vgl. hierzu auch den Beitrag von Nehls in diesem Heft).

Vor allem in der Orientierung an Lernergebnissen und nicht an Abschlüssen sowie aufgrund der Möglichkeit, auch einzelne Kompetenzen zu zertifizieren, wurde eine Gefährdung der Beruflichkeit vermutet. So ganz ist die Aufregung nicht zu verstehen. Erstens zielt die deutsche Berufsausbildung mit dem Ziel der „beruflichen Handlungsfähigkeit“ auf Lernergebnisse; in den praxisorientierten Prüfungen werden auch nicht Kenntnisse abgefragt, sondern die Kompetenzen in konkreten Arbeitsprozessen dokumentiert. Zweitens existiert mit den externen Prüfungen ein Instrument der formellen Anerkennung informell erworbener Kompetenzen, das 2010 zum Beispiel von rund 25.000 Personen genutzt wurde (Schreiber/Gutschow 2013). Drittens ergibt sich aus der Beschreibung einzelner Kompetenzen nicht zwangsläufig eine Modularisierung der Ausbildung. Über die Ganzheitlichkeit von Berufsausbildungen wird gesondert entschieden. Viertens wird der EQR - wie wir es jetzt in den Entwürfen der nationalen Qualifikationsrahmen erkennen - in den meisten Ländern auch nicht wörtlich genommen. Den einzelnen Niveaus werden nämlich in der Mehrzahl der europäische Länder vor allem Qualifikationsabschlüsse, also In- und Outputs, zugerechnet. Das gilt vor allem für die akademischen Berufsabschlüsse, die in allen europäischen Ländern den Stufen 6 (Bachelor), 7 (Master) und 8 (Promotion) ohne weitere Hinterfragung der tatsächlichen Kompetenz zugeordnet werden. Der Verkauf von Studiengängen über hohe Studiengebühren, also von standardisierten In- und Outputs, ist zudem ein international so bedeutender Wirtschaftszweig geworden, dessen Ertragskraft man nicht durch eine Orientierung auf Lernergebnisse schmälern lassen will.

Angesichts der großen Unterschiede in den nationalen Bildungs- und Beschäftigungssystemen müssen die Leitbegriffe der europäischen Beschäftigungsstrategie und Bildungspolitik vage bleiben. Dies bietet die Möglichkeit, sie auf nationaler Ebene selbst auszulegen. Anstatt die neue europäische Begrifflichkeit offensiv mit eigenen Inhalten $\mathrm{zu}$ besetzen, stehen sich jedoch viele deutsche Berufsforscher - wie etwa Greinert (2008) - mit universalistischen Begriffsdefinitionen, in denen eine Unvereinbarkeit der europäischen Konzepte mit dem Berufsbegriff postuliert wird, selbst im Wege. Dabei lässt sich gut begründen, dass berufliche Arbeitsmärkte die besten Garanten von Flexicurity und Employability sind, da sie den Unternehmen Flexibilität und den Beschäftigten durch transferierbare Qualifikationen ein hohes Maß an Beschäftigungssicherheit bieten. Zudem war das duale Lernen durch die betriebliche Einbindung und die Orientierung an realen Prozessen schon lange vor den europäischen Beschlüssen zum EQR an Lernergebnissen orientiert. Und die beruflichen Zertifikate dokumentieren durch die handlungsgeleiteten Prüfungen nicht nur Kenntnisse, sondern auch berufliche Handlungsfähigkeit.

\section{Facharbeit im Strukturwandel}

Entgegen allen Untergangsszenarien zum Ende des Berufs ist der Anteil der beruflich Qualifizierten in Deutschland so stark wie in kaum einem anderen Land von $29 \%$ in den Jahren 1964/65 (Geißler 2002, S. 339) auf 66,6 \% im 
ABB. 2

\section{Interne, berufliche und unstrukturierte Arbeitsmärkte und berufliche Bildung 1995 - 2011}

Angaben in Prozent

ohne Berufsausbildung

Berufsausbildung

Fachhochschule/Universität

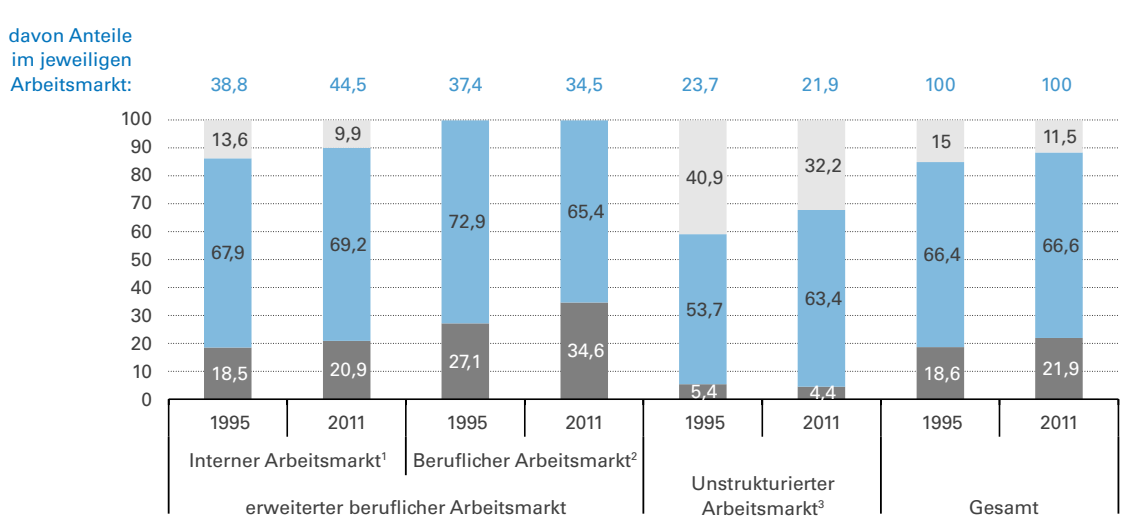

1) Betriebszugehörigkeit > als 10 Jahre

2) Ausbildung oder Studium und Betriebszugehörigkeit < als 10 Jahre.

3) Betriebszugehörigkeit < als 10 Jahre und einfache Tätigkeit.

Quelle: Berechnung und Darstellung des Autors auf Basis von Kalina (2012 und einer Neuberechnung auf Basis des SOEP (2013). ment vorbehalten sind (Voss-Dahm 2009). Auch für die Beschäftigten waren die breiteren Berufsbilder von Vorteil. Die Berufsmobilität, also der Wechsel in fachfremde Tätigkeiten, hat sich seit den 1980er Jahren nicht wesentlich verändert (Möller/Paulus 2010, S. 20). Angesichts des raschen Strukturwandels lässt sich dies nur mit den breiteren Berufsbildern erklären. Viele Tätigkeiten, die in den alten Spezialberufen zu einem Berufswechsel geführt hätten, gehören mittlerweile zum Einsatzbereich der neuen Kernberufe.

Diese Entwicklung lässt sich in allen Segmenten des deutschen Arbeitsmarktes beobachten. Im internen Arbeitsmarktsegment, das alle Beschäftigten mit einer Betriebszugehörigkeit von mehr als zehn Jahren umfasst, werden fast nur noch beruflich und akademisch Qualifizierte eingesetzt. Gleichzeitig haben durch häufigere interne Arbeitsplatzwechsel und eine Anreicherung der Tätigkeiten die Gefahren einer Dequalifikation in internen Arbeitsmärkten abgenommen. Anders als in vielen Nachbarländern mit ihren weiterhin hohen Anteilen An-und Ungelernter kann man daher in Deutschland die internen Arbeitsmärkte mittlerweile zum erweiterten beruflichen Arbeitsmarktsegment hinzuzählen. Die Unternehmen versuchen zudem, ihre qualifizierten Stammbelegschaften an sich zu binden, sodass der Anteil des internen Segments an allen Beschäftigten von 38,8 \% im Jahre 1995 auf 44,5\% gewachsen ist (Abbildung 2).

Das engere berufliche Segment umfasst per definitionem nur Fachkräfte. Hierzu zählen alle Beschäftigten mit einer Ausbildung, die auch eine Tätigkeit als Fachkraft ausüben und weniger als zehn Jahre im Unternehmen beschäftigt sind. Hier verzeichnen wir eine leichte Zunahme der Akademiker. Durch die wachsende Betriebsbindung der Fachkräfte im internen Segment hat sich der Anteil leicht verringert. Die beiden beruflichen Arbeitsmarktsegmente umfassen, wenn man die Restbestände einfacher Arbeit in internen Arbeitsmärkten abzieht, inzwischen rund $75 \%$ aller Beschäftigten.

Selbst im unstrukturierten Arbeitsmarktsegment, das Beschäftigte auf einfachen Tätigkeiten mit einer Betriebszugehörigkeit von weniger als zehn Jahren umfasst, werden zunehmend qualifizierte Kräfte eingesetzt. Durch die Verdrängung der An- und Ungelernten im unstrukturierten Arbeitsmarkt haben sich die Beschäftigungsrisiken dieser Gruppe deutlich erhöht. Dies zeigt sich auch an ihrer Arbeitslosenquote, die seit 20 Jahren über $20 \%$ liegt. Die einfache „Muskelarbeit“, die in der Vergangenheit als Prototyp der Arbeit in unstrukturierten Arbeitsmärkten galt, ist weitgehend verschwunden. Als Folge der Technisierung erfordert heute auch einfache Industriearbeit den Umgang mit abstrakten Symbolen, etwa in einem automatisierten Lager, und selbst einfache Dienstleistungsarbeit setzt hohe Kommunikationsfähigkeit voraus (Bosch/ Weinkopf 2011).

Eine Aufteilung nach Branchen, die hier nicht im Detail erfolgen kann, zeigt eine starke Abnahme unstrukturierter 
Arbeitsmärkte in den meisten Branchen des sekundären und tertiären Sektors. Hohe Anteile unstrukturierter Arbeitsmärkte finden sich noch im Nahrungsmittelgewerbe (28,8\%), einigen Teilbranchen der chemischen Industrie (Gummi und Kunststoff sowie Glas/Keramik) (26,9\%) und einigen Dienstleistungsbranchen, wie den Dienstleistungen für Unternehmen, die die Leiharbeit einschließen (31,2\%), dem Einzelhandel (42\%) und dem Gastgewerbe (59,2\%). In allen anderen Bereichen überwiegen die beruflich geprägten internen und die beruflichen Arbeitsmärkte. Wachsende Anteile beruflicher Arbeitsmärkte finden sich vor allem in Bereichen mit hoher zwischenbetrieblicher Mobilität, wie in den Bereichen Datenverarbeitung, Forschung und Entwicklung (63,3 \%), der Bauwirtschaft (45,8 \%), den Kreditinstituten (43,1 \%) und dem Maschinenbau (36,5\%) (Kalina 2012).

Problematisch für die Funktionsweise beruflicher Arbeitsmärkte ist die Verdrängung von Absolventen der beruflichen Bildung in die schlecht bezahlten sekundären Segmente der internen und beruflichen Arbeitsmärkte, in denen Niedriglöhne gezahlt werden (ebd.). Die Ausbreitung von Niedriglöhnen auch für qualifizierte Beschäftigte, darunter auch Akademiker, ist auf eine Erosion der Flächentarife, die wichtige Stützpfeiler beruflicher Arbeitsmärkte sind, zurückzuführen. In den weitgehend tariffreien Zonen des Arbeitsmarktes, wie im Gastgewerbe, sinkt hingegen nicht nur die Ausbildungsbereitschaft, sodass es überdurchschnittlich viele unbesetzte Ausbildungsplätze gibt. Auch die Abbruchquoten während der Berufsausbildung und die Abwanderungsneigung nach abgeschlossener Ausbildung sind überdurchschnittlich hoch (BiBB 2013, S. 188). In einigen Fachkräftebranchen (z. B. Bauhauptgewerbe, Dachdecker, Maler/Lackierer, Weiterbildung, Pflege) waren die Unternehmensverbände bereit, das Tarifsystem durch Branchenmindestlöhne zu stützen und sogar auch höheren Mindestlöhnen für Fachkräfte (Bauhauptgewerbe, Maler/Lackierer und Außenreinigung) zuzustimmen, um die Branchen für qualifizierte Kräfte attraktiv zu gestalten (Bosch/Weinkopf 2012).

\section{Akademisierung und duale Beruf- lichkeit}

Trotz einer raschen Zunahme von Studierenden im letzten Jahrzehnt lag die Abschlussquote von Hochschulabsolventen in Deutschland 2010 mit $30 \%$ eines Jahrgangs unter dem Durchschnitt der OECD (39\%). Der OECD-Durchschnitt ist durch eine beispielslose Hochschulexpansion in vielen Ländern angehoben worden (OECD 2012, S. 67). Das duale System verfügt mit der Aufstiegsfortbildung über eine Oberetage qualifizierter Fortbildung, die im Niveau vielen beruflich orientierten Bachelorabschlüssen in anderen Ländern überlegen sind. Wenn man die Abschlüsse des
Tertiärbereichs B einbezieht, die praktischer orientiert sind und u.a. Meister, Fachwirte und Techniker umfassen, dann schrumpft der Abstand der Tertiärabschlüsse zum OECDDurchschnitt, bleibt aber weiterhin bestehen (Müller 2009). Die OECD und mittlerweile die meisten Bildungspolitiker in Deutschland sehen die deutsche Wettbewerbsfähigkeit grundlegend gefährdet, wenn Deutschland nicht mindestens den OECD-Durchschnitt bei den Hochschulabsolventen erreicht. Dahinter steht auch die These, dass man mehr theoretisch Qualifizierte braucht, die das duale System nicht hervorbringen kann.

Höhere Akademikerzahlen in einem Land signalisieren allerdings nur dann eine höhere Leistungsfähigkeit, wenn die Abschlüsse auch mit höheren Kompetenzniveaus als bei einer Berufsausbildung verbunden sind, es auch hinreichend Arbeitsplätze gibt, auf denen ein akademisches Kompetenzniveau abgerufen wird, und wenn der Kompetenzgewinn an der Spitze nicht mit Kompetenzverlusten auf der mittleren Qualifikationsebene teuer bezahlt werden muss. Deswegen gibt es mehrere Gründe, eine einseitige Orientierung auf die Hochschulausbildung zu hinterfragen.

Erstens liegt in allen entwickelten OECD-Ländern der Anteil der Beschäftigungsverhältnisse mit hochqualifizierten Tätigkeiten zwischen 15 und maximal $25 \%$ (Müller 2009). Die Arbeitsplatzstruktur ist somit erheblicher konservativer, als es die Thesen zur Wissensgesellschaft erwarten lassen, und sie erfordert nur einen leichten Ausbau der Hochschulbildung. Bildet man mehr Akademiker aus als von der Nachfrageseite gefordert, steigt das Risiko von Dequalifikation und unterwertiger Beschäftigung, das wir in Ländern mit hohen Akademikeranteilen schon beobachten können (z. B. Anderson 2009). So waren zum Beispiel $200737 \%$ der kanadischen, 33 \% der USamerikanischen und $44 \%$ der spanischen Hochschulabsolventen zwischen 25 und 29 Jahren in Tätigkeiten unterhalb des akademischen Niveaus, also in den Berufsgruppen 4 bis 9 der „Internationalen Standardklassifikation der Berufe“ (ISCO), tätig gegenüber $20 \%$ in Deutschland (OECD 2010: Indicator C3.7).

Zweitens kommt der Druck zum Ausbau der Hochschulen in den meisten Ländern nicht vom Arbeitsmarkt, sondern von den Jugendlichen und ihren Eltern. Dieser Druck ist umso stärker, je mehr der Zugang zu interessanten und gutbezahlten Tätigkeiten akademisch Qualifizierten vorbehalten ist. Durch den Mangel an attraktiven beruflichen Ausbildungswegen kommt es in vielen Ländern zu künstlich aufgeblähten Akademikerzahlen. Alle Länder mit hohen Abschlussquoten im tertiären Bereich haben wenig entwickelte berufliche Bildungssysteme. Das freiwerdende Terrain wurde von der akademischen Ausbildung übernommen. Zum Teil wandert die klassische Berufsbildung in den tertiären Bereich ab, um attraktiv zu bleiben. Viele Bachelorstudiengänge etwa in Kanada, Australien oder den USA ähneln einer schulischen Berufsausbildung in Deutschland (Bosch/Charest 2010). 
Drittens folgt aus unterdurchschnittlichen Akademikeranteilen nicht notwendigerweise eine Kompetenzlücke. Ein Vergleich der Kompetenzniveaus Erwachsener zwischen 25 und 64 Jahren in den USA und in Deutschland auf der Basis der Ergebnisse des International Adult Literacy Survey (IALS) zeigt, dass die Kompetenzniveaus der beruflich qualifizierten Deutschen im Durchschnitt nahe an die der Akademiker in den USA reichen und dass der Anteil der Personen mit hohen Kompetenzen in beiden Ländern in etwa gleich ist (Anger/Plünnecke 2009).

Viertens wurde beim Kompetenzvergleich noch nicht berücksichtigt, dass die IALS nur Kenntnisse misst, nicht aber berufliche Handlungsfähigkeit, was ja als die zentrale Stärke dualer Berufsausbildung gilt. Es spricht einiges dafür, dass in der engen Kooperation von akademisch qualifizierten Entwicklern und Führungskräften mit qualifizierten Machern das Geheimnis der deutschen Wettbewerbsfähigkeit liegt. In mehreren, leider schon etwas älteren vergleichenden Untersuchungen zu deutschen, britischen und US-amerikanischen Unternehmen mit gleichen Produkten und Technologien wurden erhebliche Produktivitätsvorteile in deutschen Betrieben festgestellt (z. B. Wagner/Finegold 1997). In den Vergleichsländern USA und Großbritannien wird die mittlere Führungsebene mit Hochschulabsolventen besetzt, die für diese Funktionen durch ihre breite theoretische Ausbildung überqualifiziert und mit ihrem Mangel an praktischem Umsetzungswissen gleichzeitig unterqualifiziert sind.

Der letzte Punkt wirft die Frage auf, ob Erfahrungslernen durch die Akademisierung der Ausbildung an Bedeutung verlieren wird. Der von Baethge et al. (2007) unterstrichene Bedeutungszuwachs von systematischem Wissen lässt sich kaum bestreiten. In der Berufsausbildung hat dies in der besseren schulischen Vorbildung der Auszubildenden und neuen Lehrinhalten seinen Niederschlag gefunden. Gleichzeitig wissen wir aber auch, dass berufliche Handlungsfähigkeit erst durch die Fähigkeit zur Re-Kontextualisierung des systematischen Wissens erworben wird, was man nur in realen Arbeitsprozessen lernt. Dies gilt auch für den Erwerb des in innovativen Arbeitsprozessen entstehenden und noch nicht kodierten informellen Wissens (Pfeiffer 2012).

Die deutschen Unternehmen stemmen sich gegen den Verlust an erfahrungsgebundenem Lernen durch die Hochschulexpansion. Zwar hat sich in den letzten Jahren der Anteil der Führungskräfte mit einem Studium auf Kosten der rein beruflich Qualifizierten deutlich erhöht. Franz/ Voss-Dahm (2011) zeigen jedoch, dass die meisten zuvor eine berufliche Ausbildung erworben haben und oft erst durch die Perspektive eines betrieblichen Aufstiegs zum Studium ermuntert worden sind. Gleichzeitig sind in den letzten Jahren neue duale Studiengänge vielfach auf Initiative von Unternehmen entstanden, die Studium und duale berufliche Bildung kombinieren (vgl. den Beitrag von Krone/Mill in diesem Heft). Die Vorteile von Hochschul- und Berufsausbildung sollen in diesem neuen Hybridmodell der beruflichen Bildung kombiniert werden.

\section{Fazit}

Angesichts der hohen Reformfähigkeit des dualen Systems der Berufsausbildung sowie der stabilen Interessen der Unternehmen am Einsatz der von ihnen selbst ausgebildeten Fachkräfte erscheinen die Thesen zur Krise des Berufs im Nachhinein „nicht nur als Überspitzung, sondern als eine eklatante historische Fehleinschätzung" (Möller/Paulus 2010, S. 19). Die modernisierte Berufsausbildung in breiten Berufen sozialisiert die Auszubildenden heute immer weniger auf hierarchische Formen der Arbeitsorganisation mit engem fachlichen Zuschnitt, sondern immer mehr auf die Selbstorganisation von Teams mit unterschiedlichen Berufen in flexiblen Formen der Arbeitsorganisation. Daher ist kaum nachvollziehbar, dass ausgerechnet die Auflösung des Taylorismus mit seinen engen Einsatzfeldern eine Krise der Berufsausbildung auslösen sollte (Baethge/ Baethge-Kinsky 1998).

Berufliche Arbeitsmärkte bieten hohen Schutz von Beschäftigten auch bei zwischenbetrieblicher Mobilität (Flexicurity) und stärken damit die Beschäftigungsfähigkeit. Die Unternehmen in Deutschland haben im Übergang zu flexibleren Organisationsformen seit 1990 erfolgreich auf die Überschussqualifikationen von Fachkräften gesetzt, während in vielen anderen Ländern polarisierte Qualifikationsstrukturen mit hohen Akademikeranteilen auf der einen und hohen Anteilen von An- und Ungelernten auf der anderen Seite dominieren. Mittlerweile sind rund $75 \%$ der deutschen Beschäftigten in beruflichen Arbeitsmärkten tätig, haben also eine abgeschlossene Berufs- oder Hochschulausbildung und üben eine qualifizierte Tätigkeit aus. Die Kombination von gut ausgebildeten Akademikern, einer dual ausgebildeten mittleren Führungsschicht und beruflich Qualifizierten mit hoher Autonomie ist das Geheimnis der deutschen Wettbewerbsfähigkeit. Durch das duale Studium versuchen viele Unternehmen, die Vorteile betrieblichen Lernens und akademischer Ausbildung gerade für ihre mittlere Führungsebene zu kombinieren.

Die hohen Akademikeranteile in anderen Ländern sind kein sinnvoller Orientierungspunkt für die deutsche Bildungspolitik. Sie haben nicht zu Kompetenzvorsprüngen geführt, sondern sind Folge eines Reputationsverlusts der beruflichen Bildung in diesen Ländern, die von ihren Akteuren nicht "gepflegt“ und aufgewertet worden ist. Die Expansion der Hochschulbildung ist in diesen Ländern nicht durch die Entwicklung der Arbeitsplatzstruktur getrieben, sondern durch den Wunsch der Eltern und der Jugendlichen, sich im Wettbewerb um gute Arbeitsplätze einen Startvorteil zu verschaffen.

Eine schleichende Erosion des Berufsbildungssystems durch eine einseitige Akademisierung zu vermeiden, ist eine der zentralen bildungspolitischen Herausforderungen der nächsten Jahre. Der deutsche „Sonderweg“, im nationalen Qualifikationsrahmen die Aufstiegsfortbildungen mit dem Bachelor und Master gleichzustellen, ist ein wichtiger 
Ansatzpunkt. Eine zweite Herausforderung liegt in der lohnpolitischen Unterstützung der Berufsbildung. Durch die Erosion der Tarifbindung in vielen Bereichen der deutschen Wirtschaft werden viele beruflich Qualifizierte in den Niedriglohnsektor abgedrängt. Dies beeinträchtigt auf Dauer die Ausbildungsbereitschaft. Notwendig sind Maßnahmen zur Stabilisierung der Tarifsysteme, wie Mindestlöhne und allgemeinverbindliche $\mathrm{Ta}$ rifverträge.

\section{LITERATUR}

Anderson, P. (2009): Intermediate occupations and the conceptual and empirical limitations of the hourglass economy thesis, in: Work, Employment and Society 23 (1), S. 169-180

Anger, C./Plünnecke, A. (2009): Signalisiert die Akademikerlücke eine Lücke bei den Hochqualifizierten? Deutschland und die USA im Vergleich: Institut der Deutschen Wirtschaft, IWTrends 3/2009, Köln

Baethge, M./Baethge-Kinsky, V. (1998): Jenseits von Beruf und Beruflichkeit? Neue Formen von Arbeitsorganisation und Beschäftigung und ihre Bedeutung für eine zentrale Kategorie gesellschaftlicher Integration, in: Mitteilungen aus der Arbeitsmarkt- und Berufsforschung (MITTAB) 31 (Sonderdruck), Nürnberg, S. $461-472$

Baethge, M./Solga, H./Wieck, M. (unter Mitarbeit von Christiane Petsch) (2007): Berufsbildung im Umbruch. Signale eines überfälligen Aufbruchs, Gutachten zur beruflichen Bildung in Deutschland im Auftrag der Friedrich-EbertStiftung

Bekker, S./Wilthagen, T. (2008): Flexicurity - a European Approach to Labour Market Policy, in: Intereconomics March/April, S. 68-73

Böhle, F./Altmann, N. (1972): Industrielle Arbeit und soziale Sicherheit. Eine Studie über die Risiken im Arbeitsprozeß und auf dem Arbeitsmarkt, Frankfurt a. M.

Bosch, G. (1978): Arbeitsplatzverlust: Soziale Folgen einer Betriebsstillegung, Frankfurt a. M./New York

Bosch, G. (2010): Zur Zukunft der dualen Berufsausbildung in Deutschland, in: Bosch, G./Krone, S./Langer, D. (Hrsg.): Das Berufsbildungssystem in DeutschIand: Aktuelle Entwicklungen und Standpunkte, Wiesbaden, S. 37-61 Bosch, G./Charest, J. (Hrsg.) (2010): Vocational Training - International Perspectives, London

Bosch, G./Weinkopf, C. (2011): „Einfacharbeit“ im Dienstleistungssektor, in: Arbeit 20 (3), S. 173-187

Bosch, G./Weinkopf, C. (2012): Wirkungen der Mindestlohnregelungen in acht Branchen. Expertise im Auftrag der Abteilung Wirtschafts- und Sozialpolitik der Friedrich-Ebert-Stiftung, WISO Diskurs November, Bonn

Brettschneider, M./Schwarz, H. (2011): Berufsbildung in Unordnung? Strukturierung von Ausbildung, in: BWP 2/2011, S. 43-46

Brötz, R. (2009): Sind 350 Berufe zuviel? Identifizierung und Schaffung von Berufsfamilien, Didacta - die Bildungsmesse, 13. Februar, Köln

Brötz, R./Schapel-Kaiser, F./Schwarz, H. (2008): Berufsfamilien als Beitrag zur Stärkung des Berufsprinzips, in: BWP 4/2008, S. 23-26

Brussig, M./Knuth, M. (2009): Individuelle Beschäftigungsfähigkeit: Konzept, Operationalisierung und erste Ergebnisse, in: WSI-Mitteilungen 62 (6),

S. 287-294, http://www.boeckler.de/cps/rde/xbcr/hbs/wsimit_2009_06_brussig. pdf

Bundesinstitut für Berufsbildung (BiBB) (2013): Datenreport zum Berufsbildungsbericht 2013, Bonn

Daheim, H. (1967): Der Beruf in der modernen Gesellschaft, Köln/Berlin

Drexel, I. (1980): Die Krise der Anlernung im Arbeitsprozess, in:

Soziale Welt 31 (3), S. 368-395

Franz, C./Voss-Dahm, D. (2011): Ohne Studium (k)eine Führungsposition? Nach wie vor starke Bedeutung von beruflichen Bildungsabschlüssen bei Führungskräften in der Privatwirtschaft, IAQ-Report 2/2011, Duisburg/Essen Geißler, R. (2002): Die Sozialstruktur Deutschlands, Wiesbaden Greinert, W.-D. (2008): Beschäftigungsfähigkeit und Beruflichkeit - zwei konkurrierende Modelle der Erwerbsqualifizierung?, in: BWP 4/2008, S. 9-12 Hahne, K. (1999): Weiterentwicklung des auftragsorientierten Lernens im Handwerk durch Lernortkooperation mit Auftragstypen-Leitfäden, in: Jennewein, K. (Hrsg.): Lernen und Arbeiten in der dualen Berufsbildung, Bremen, S. $201-226$
Hartmann, H. (1968): Arbeit, Beruf, Professionen, in: Soziale Welt 19 (3/4), S. $193-216$

Heidegger G./Rauner, F. (1997): Reformbedarf in der beruflichen Bildung, Gutachten für das Ministerium für Wirtschaft und Mittelstand, Technologie und Verkehr des Landes NRW, Düsseldorf

Kalina, T. (2012): Niedriglohnbeschäftigte in der Sackgasse? - Was die Segmentationstheorie zum Verständnis des Niedriglohnsektors in Deutschland beitragen kann, Dissertation, Duisburg/Essen

Lipsmeier, A. (1998): Vom verblassenden Wert des Berufs für das berufliche Lernen, in: Zeitschrift für Berufs- und Wirtschaftspädagogik 94 (4), S. 481-495 Mangum, G. L. (1976): Employability, employment and income. A reassessment of manpower policy, Salt Lake City

McKenzie, P./Wurzburg, G. (1998): Lifelong learning and Employability, in:The OECD Observer (209) Dezember 1997/Januar 1998, S. 13-17

Möller, J./Paulus, W. (2010): Perspektiven einer modernen Berufsforschung, in Euler, D./Walwei, U./Weiß, R. (Hrsg.) (2010): Berufsforschung für eine moderne Berufsausbildung - Stand und Perspektiven, Zeitschrift für Berufs- und Wirtschaftspädagogik, Beiheft 24, S. 11-35

Müller, N. (2009): Akademikerausbildung in Deutschland: Blinde Flecken beim internationalen OECD-Vergleich, in: BWP 2/2009, S. 42-46

o. A. (1983): Experiments with the first year of apprenticeships, Vol. 3: British Ministry of Education, London

Organisation for Economic Co-operation and Development (OECD) (2010): Education at a glance 2010, Paris

Organisation for Economic Co-operation and Development (OECD) (2012): Education at a glance 2012, Paris

Pahl, J.-P. (2010): Fachschule:Theorie und Praxis einer beruflichen Weiterbildungseinrichtung: Bertelsmann-Stiftung, Bielefeld

Petersen, A. W. (2012): Struktur- und Profilwandel der Elektro- und IT-Ausbildungsberufe als systemischer Gegenstand der Berufs- und Berufsbildungsforschung: Berufsbildungsinstitut Arbeit und Technik, Universität Flensburg Pfeiffer, S. (2012): Wissenschaftliches Wissen und Erfahrungswissen, ihre Bedeutung in innovativen Unternehmen und was das mit (beruflicher) Bildung zu tun hat, in: Kuda, E./Kaßebaum, B./Spöttl, G./Strauß, J. (Hrsg.): Akademisierung der Arbeit: Hat berufliche Bildung noch eine Zukunft? Hamburg, S. $203-219$

Schumann, M. (2002): Struktureller Wandel und Entwicklung der Qualifikationsanforderungen, Vortrag auf dem 4. BiBB-Fachkongress 23.-25.10.2002, Berlin

Schreiber, D./Gutschow, K. (2013): Externen Prüfungsteilnehmern auf der Spur. Wie holen jüngere Erwachsene einen Berufsabschluss nach?, BiBB-Report 20/13, Bonn

Sengenberger, W. (1987): Struktur und Funktionsweise von Arbeitsmärkten. Die Bundesrepublik Deutschland im internationalen Vergleich, Frankfurt a. M./ New York

Spöttl, G./Blings, J. (2011): Kernberufe. Ein Baustein für ein transnationales Berufsbildungskonzept, Berufliche Bildung in Forschung, Schule und Arbeitswelt, Bd. 6, Frankfurt a. M.

Ulrich, E./Lahner, M. (1970): Zur Prognose „neuer Berufe“, in: MittAB 3 (1) S. 33-44, Nürnberg

Voss, G. G. (1998): Die Entgrenzung von Arbeit und Arbeitskraft. Eine subjektorientierte Interpretation des Wandels der Arbeit, in: MittAB 31 (3), S. 473-487 Voss-Dahm, D. (2009): Über die Stabilität sozialer Ungleichheit im Betrieb. Verkaufsarbeit im Betrieb, Verkaufsarbeit im Einzelhandel, Berlin

Wagner, K./Finegold, D. (1997): Der Einfluß der Aus- und Weiterbildung auf die Arbeitsorganisation - Eine Untersuchung in der Fertigung US-Amerikanischer Maschinenbauunternehmen, in: Clermont, A./Schmeisser, W. (Hrsg.): Internationales Personalmanagement, München, S. 147-164

\section{AUTOR}

GERHARD BOSCH, Prof. Dr., ist Geschäftsführender Direktor des Institut Arbeit und Qualifikation (IAQ) an der Universität Duisburg-Essen. Arbeitsschwerpunkte: Arbeitsmarkt- und Strukturpolitik, Arbeitszeit, Personalpolitik.

gerhard.bosch@uni-due.de 